Pré-Publicações do Departamento de Matemática

Universidade de Coimbra

Preprint Number 06-38

\title{
NON-SMOOTH ATOMIC DECOMPOSITIONS OF ANISOTROPIC FUNCTION SPACES AND SOME APPLICATIONS
}

\author{
SUSANA D. MOURA, IWONA PIOTROWSKA AND MARIUSZ PIOTROWSKI \\ Abstract: The main purpose of the present paper is to extend the theory of \\ non-smooth atomic decompositions to anisotropic function spaces of Besov and \\ Triebel-Lizorkin type. Moreover, the detailed analysis of the anisotropic homogene- \\ ity property is carried out. We also present some results on pointwise multipliers \\ in special anisotropic function spaces.
}

KEYWORDS: anisotropic function spaces, non-smooth atoms, pointwise multipliers. AMS Subject Classification (2000): 42B35, 46E35, 28 A80.

\section{Introduction}

In recent years, many efforts have been made to develop decomposition techniques in function spaces using atoms, quarks or wavelets as building blocks. All these techniques have found widespread applications in other branches of the theory of function spaces and still remain very much alive as subjects of current research. For a deeper discussion of these techniques, the reader is referred to the recent monograph [13].

In the present paper we are concerned with non-smooth atomic decompositions of special anisotropic function spaces of Besov type. Using these non-smooth atoms one can also improve the smoothness assumptions for classical smooth anisotropic atoms according to Farkas [3] in a natural way. The problem of extending the theory of non-smooth isotropic atoms to the anisotropic case was posed by $\mathrm{H}$. Triebel in [13, Remark 5.16]. The second purpose of this work is to study pointwise multipliers in these function spaces. We now describe briefly the contents of the paper. In Section 2 we set up notation and terminology and summarize some basic facts on anisotropic function spaces. In Section 3 the homogeneity properties of anisotropic function spaces are presented. Section 4 is concerned with the non-smooth atomic decomposition in some anisotropic spaces of Besov type. These results are

\footnotetext{
Received August 30, 2006.

This research was supported by CMUC, the second-named author was supported by Junior Research Team Fractal analysis.
} 
used in Section 5 to obtain some new assertions on pointwise multipliers in anisotropic function spaces.

\section{Preliminaries}

2.1. Notation and Conventions. For a real number $a$, let $a_{+}:=\max (a, 0)$. By $c, c_{1}, c_{2}$, etc. we denote positive constants independent of appropriate quantities. For two non-negative expressions (i.e. functions or functionals) $\mathcal{A}, \mathcal{B}$, the symbol $\mathcal{A} \lesssim \mathcal{B}$ (or $\mathcal{A} \gtrsim \mathcal{B}$ ) means that $\mathcal{A} \leq c \mathcal{B}$ (or $c \mathcal{A} \geq \mathcal{B}$ ). If $\mathcal{A} \lesssim \mathcal{B}$ and $\mathcal{A} \gtrsim \mathcal{B}$, we write $\mathcal{A} \sim \mathcal{B}$ and say that $\mathcal{A}$ and $\mathcal{B}$ are equivalent. For $p \in[1, \infty]$, the conjugate number $p^{\prime}$ is defined by $1 / p+1 / p^{\prime}=1$ with the convention that $1 / \infty=0$. Given two quasi-Banach spaces $X$ and $Y$, we write $X \hookrightarrow Y$ if $X \subset Y$ and the natural embedding is bounded. In the following let both $\mathrm{d} x$ and $|\cdot|$ stand for the Lebesgue measure in $\mathbb{R}^{n}$. Let

$$
\left(\Delta_{h}^{1} f\right)(x)=f(x+h)-f(x), \quad\left(\Delta_{h}^{m+1} f\right)(x)=\Delta_{h}^{1}\left(\Delta_{h}^{m} f\right)(x)
$$

with $x, h \in \mathbb{R}^{n}$ and $m \in \mathbb{N}$ be the iterated differences in $\mathbb{R}^{n}$. For $x \in \mathbb{R}^{n}$ and $\beta, \gamma \in \mathbb{N}_{0}^{n}$ we put

$$
\beta \gamma=\gamma \beta=\sum_{j=1}^{n} \gamma_{j} \beta_{j} \quad \text { and } \quad x^{\gamma}=x_{1}^{\gamma_{1}} \cdot \ldots \cdot x_{n}^{\gamma_{n}} .
$$

Let $\mathcal{S}\left(\mathbb{R}^{n}\right)$ stand for the Schwartz space of all complex-valued rapidly decreasing $C^{\infty}$ functions on $\mathbb{R}^{n}$. Further, we denote by $\mathcal{S}^{\prime}\left(\mathbb{R}^{n}\right)$ its topological dual, the space of all tempered distributions.

2.2. Anisotropic function spaces. In this subsection we introduce the anisotropic Besov and Triebel-Lizorkin spaces and describe some important properties. Let us start by recalling briefly the basic ingredients needed to introduce these spaces by the Fourier-analytical approach. Throughout the paper we call the vector

$$
\alpha=\left(\alpha_{1}, \ldots, \alpha_{n}\right) \quad \text { with } \quad 0<\alpha_{1} \leq \ldots \leq \alpha_{n}<\infty \quad \text { and } \quad \sum_{j=1}^{n} \alpha_{j}=n
$$

an anisotropy in $\mathbb{R}^{n}$. For $t>0, r \in \mathbb{R}$ and $x=\left(x_{1}, \ldots, x_{n}\right) \in \mathbb{R}^{n}$ we put

$$
t^{\alpha} x:=\left(t^{\alpha_{1}} x_{1}, \ldots t^{\alpha_{n}} x_{n}\right) \text { and } t^{r \alpha} x:=\left(t^{r}\right)^{\alpha} x
$$


For $x=\left(x_{1}, \ldots, x_{n}\right) \in \mathbb{R}^{n}, x \neq 0$, let $|x|_{\alpha}$ be the unique positive number $t$ such that

$$
\frac{x_{1}^{2}}{t^{2 \alpha_{1}}}+\ldots+\frac{x_{n}^{2}}{t^{2 \alpha_{n}}}=1
$$

and put $|0|_{\alpha}=0$. It turns out that $|\cdot|_{\alpha}$ is an anisotropic distance function according to [3, Definition 2.1] in $C^{\infty}\left(\mathbb{R}^{n}\right) \backslash\{0\}$. Note that in the isotropic case, which means $\alpha_{1}=\cdots=\alpha_{n}=1,|x|_{\alpha}$ is the Euclidean distance of $x$ to the origin.

Let $\varphi^{\alpha} \in \mathcal{S}\left(\mathbb{R}^{n}\right)$ be a function such that

$$
\varphi^{\alpha}(x)=1 \quad \text { for } \quad|x|_{\alpha} \leq 1 \quad \text { and } \quad \operatorname{supp} \varphi^{\alpha} \subset\left\{x \in \mathbb{R}^{n}:|x|_{\alpha} \leq 2\right\} .
$$

For each $j \in \mathbb{N}$ we define

$$
\varphi_{j}^{\alpha}(x):=\varphi^{\alpha}\left(2^{-j \alpha} x\right)-\varphi^{\alpha}\left(2^{-(j-1) \alpha} x\right), \quad x \in \mathbb{R}^{n},
$$

and put $\varphi_{0}^{\alpha}=\varphi^{\alpha}$. Then since $\sum_{j=0}^{\infty} \varphi_{j}^{\alpha}(x)=1$ for all $x \in \mathbb{R}^{n}$, the sequence $\left(\varphi_{j}^{\alpha}\right)_{j \in \mathbb{N}_{0}}$ is an anisotropic resolution of unity. Recall that $\left(\varphi_{j}^{\alpha} \widehat{f}\right)^{\vee}$ is an entire function on $\mathbb{R}^{n}$.

Definition 2.1. Let $\alpha$ be an anisotropy as in (2) and let $\varphi^{\alpha}=\left(\varphi_{j}^{\alpha}\right)_{j \in \mathbb{N}_{0}}$ be an anisotropic dyadic resolution of unity in the sense of (5).

(i) For $0<p, q \leq \infty$ and $s \in \mathbb{R}$ the anisotropic Besov space $B_{p q}^{s, \alpha}\left(\mathbb{R}^{n}\right)$ is defined to be the set of all tempered distributions $f \in \mathcal{S}^{\prime}\left(\mathbb{R}^{n}\right)$ such that

$$
\left\|f \mid B_{p q}^{s, \alpha}\left(\mathbb{R}^{n}\right)\right\|:=\left(\sum_{j=0}^{\infty} 2^{j s q}\left\|\left(\varphi_{j}^{\alpha} \widehat{f}\right)^{\vee} \mid L_{p}\left(\mathbb{R}^{n}\right)\right\|^{q}\right)^{1 / q}
$$

is finite. In the limiting case $q=\infty$ the usual modification is required.

(ii) For $0<p<\infty, 0<q \leq \infty$ and $s \in \mathbb{R}$ the anisotropic Triebel-Lizorkin space $F_{p q}^{s, \alpha}\left(\mathbb{R}^{n}\right)$ is defined to be the set of all tempered distributions $f \in$ $\mathcal{S}^{\prime}\left(\mathbb{R}^{n}\right)$ such that

$$
\left\|f\left|F_{p q}^{s, \alpha}\left(\mathbb{R}^{n}\right)\|:=\|\left(\sum_{j=0}^{\infty} 2^{j s q}\left|\left(\varphi_{j}^{\alpha} \widehat{f}\right)^{\vee}(\cdot)\right|^{q}\right)^{1 / q}\right| L_{p}\left(\mathbb{R}^{n}\right)\right\|
$$

is finite. In the limiting case $q=\infty$ the usual modification is required. 
Remark 2.2. We occasionally use the symbol $A_{p q}^{s, \alpha}\left(\mathbb{R}^{n}\right)$ to consider the spaces $B_{p q}^{s, \alpha}\left(\mathbb{R}^{n}\right)$ and $F_{p q}^{s, \alpha}\left(\mathbb{R}^{n}\right)$ simultaneously. It turns out that $A_{p q}^{s, \alpha}\left(\mathbb{R}^{n}\right)$ are quasiBanach spaces which are independent of $\varphi^{\alpha}$, in the sense of equivalent quasinorms, according to either $(6)$ or $(7)$. Taking $\alpha=(1, \ldots, 1)$ brings us back to the isotropic case usually denoted by $B_{p q}^{s}\left(\mathbb{R}^{n}\right)$ and $F_{p q}^{s}\left(\mathbb{R}^{n}\right)$. The above Fourier analytical approach to anisotropic function spaces is due to H. Triebel [9].

Let us now make a few historical comments on anisotropic function spaces. A detailed treatment of the history of anisotropic function spaces can be found in [13, Section 5]. There is quite an extensive literature concerning anisotropic function spaces, beginning with the work of S. M. Nikol'skij and O. V. Besov. The key objective is to make the smoothness properties of an element from some function space dependent on the chosen direction in $\mathbb{R}^{n}$. Roughly speaking, elements of $B_{p q}^{s, \alpha}\left(\mathbb{R}^{n}\right)$ and $F_{p q}^{s, \alpha}\left(\mathbb{R}^{n}\right)$ are smooth of order $s / \alpha_{r}$ in direction of the $r$-th coordinate with $r=1, \ldots, n$. Let us explain this relationship in detail by discussing classical anisotropic spaces. Let $1<p<\infty$ and $\bar{k}=\left(k_{1}, \ldots, k_{n}\right)$ with $k_{r} \in \mathbb{N}, r=1, \ldots, n$. The subspace of all $f \in L_{p}\left(\mathbb{R}^{n}\right)$ for which the norm

$$
\left\|f\left|W_{p}^{\bar{k}}\left(\mathbb{R}^{n}\right)\|:=\| f\right| L_{p}\left(\mathbb{R}^{n}\right)\right\|+\sum_{r=1}^{n}\left\|\frac{\partial^{k_{r}} f}{\partial x_{r}^{k_{r}}} \mid L_{p}\left(\mathbb{R}^{n}\right)\right\|
$$

is finite is called the classical anisotropic Sobolev space $W_{p}^{\bar{k}}\left(\mathbb{R}^{n}\right)$. It is easily seen that if $k_{1}=\ldots=k_{n}=k \in \mathbb{N}$, then the space $W_{p}^{\bar{k}}\left(\mathbb{R}^{n}\right)$ becomes the wellknown isotropic Sobolev space $W_{p}^{k}\left(\mathbb{R}^{n}\right)$. We now describe a generalization of classical anisotropic Sobolev spaces, replacing the smoothness vector $\bar{k}=$ $\left(k_{1}, \ldots, k_{n}\right)$ consisting only of natural numbers by the vector with real entries. We consider the anisotropic lift operator $I_{\sigma}^{\alpha}$ with $\sigma \in \mathbb{R}$, which takes $f \in$ $\mathcal{S}^{\prime}\left(\mathbb{R}^{n}\right)$ to

$$
I_{\sigma}^{\alpha}(f):=\left(\left[\sum_{r=1}^{n}\left(1+\xi_{r}^{2}\right)^{1 / 2 \alpha_{r}}\right]^{\sigma} \widehat{f}\right)^{\vee}
$$

Then we refer to

$$
H_{p}^{\bar{s}}\left(\mathbb{R}^{n}\right):=I_{-s}^{\alpha} L_{p}\left(\mathbb{R}^{n}\right)
$$

with $\bar{s}=\left(s_{1}, \ldots, s_{n}\right)$ and $s_{r}=s / \alpha_{r}, r=1, \ldots, n$, as anisotropic Sobolev spaces or anisotropic Bessel potential spaces. In addition, if $s_{r} \in \mathbb{N}$ for all 
$r=1, \ldots, n$, then

$$
H_{p}^{\bar{s}}\left(\mathbb{R}^{n}\right)=W_{p}^{\bar{s}}\left(\mathbb{R}^{n}\right)
$$

become the classical anisotropic Sobolev spaces according to (8). We proceed by describing the classical anisotropic Besov spaces. Let $1<p<\infty$ and $1 \leq q \leq \infty$. Moreover let $\bar{s}=\left(s_{1}, \ldots, s_{n}\right)$ with $0<s_{r}<M_{r} \in \mathbb{N}$ and set $\bar{M}=\left(M_{1}, \ldots, M_{n}\right)$. The classical anisotropic Besov space consists of those $f \in L_{p}\left(\mathbb{R}^{n}\right)$ for which

$$
\left\|f\left|B_{p q}^{\bar{s}}\left(\mathbb{R}^{n}\right)\left\|_{\bar{M}}:=\right\| f\right| L_{p}\left(\mathbb{R}^{n}\right)\right\|+\sum_{r=1}^{n}\left(\int_{0}^{1} t^{-s_{r} q}\left\|\Delta_{t, r}^{M_{r}} f \mid L_{p}\left(\mathbb{R}^{n}\right)\right\|^{q} \frac{\mathrm{d} t}{t}\right)^{1 / q}
$$

is finite. Here $\Delta_{t, r}^{m} f=\Delta_{h}^{m} f$ with $h=t e_{r}, t \in \mathbb{R}$ denote the iterated differences according to (1) in direction of the $r$-th coordinate and $e_{r}$ stands for the corresponding unit vector in $\mathbb{R}^{n}$. Once again putting $s_{1}=\ldots s_{n}=s>0$, we recover the classical Besov spaces as presented for instance in [10, Section 1.2.5]. We now shall discuss the relation between the function spaces introduced in Definition 2.1 and the classical anisotropic function spaces. Given an anisotropic smoothness vector $\bar{s}=\left(s_{1}, \ldots, s_{n}\right)$, we define the so-called mean smoothness $s$ and $\alpha=\left(\alpha_{1}, \ldots, \alpha_{n}\right)$ by

$$
\frac{1}{s}=\frac{1}{n} \sum_{r=1}^{n} \frac{1}{s_{r}} \text { and } \quad \alpha_{r}=\frac{s}{s_{r}}, \quad r=1, \ldots, n .
$$

This makes it possible to recover in Definition 2.1 the classical anisotropic function spaces. For instance, restricting the range of involved indices in Definition $2.1(\mathrm{i})$ to $1<p<\infty$ and $1 \leq q \leq \infty$, we obtain $B_{p q}^{\bar{s}}\left(\mathbb{R}^{n}\right)=$ $B_{p q}^{s, \alpha}\left(\mathbb{R}^{n}\right)$. On the other hand, given a function space $A_{p q}^{s, \alpha}\left(\mathbb{R}^{n}\right)$ with a suitable combination of indices, the vector $\bar{s}=\left(s_{1}, \ldots, s_{n}\right)$ is calculated by $\bar{s}=\left(s / \alpha_{1}, \ldots, s / \alpha_{n}\right)$. Let $s \in \mathbb{R}$ and $1<p<\infty$. Then it can be shown that

$$
F_{p, 2}^{s, \alpha}\left(\mathbb{R}^{n}\right)=H_{p}^{\bar{s}}\left(\mathbb{R}^{n}\right)
$$

in the sense of equivalent norms. Moreover, we have the following anisotropic Paley-Littlewood theorem

$$
F_{p, 2}^{0, \alpha}\left(\mathbb{R}^{n}\right)=L_{p}\left(\mathbb{R}^{n}\right) .
$$

We conclude this subsection by discussing some characterizations of the anisotropic spaces $B_{p q}^{s, \alpha}\left(\mathbb{R}^{n}\right)$ and $F_{p q}^{s, \alpha}\left(\mathbb{R}^{n}\right)$ with $s>\sigma_{p}$ in terms of the quasinorms of its homogeneous counterparts, denoted by $\dot{B}_{p q}^{s, \alpha}\left(\mathbb{R}^{n}\right)$ and $\dot{F}_{p q}^{s, \alpha}\left(\mathbb{R}^{n}\right)$, 
respectively. Recall that the corresponding homogeneous anisotropic spaces $\dot{B}_{p q}^{s, \alpha}\left(\mathbb{R}^{n}\right)$ and $\dot{F}_{p q}^{s, \alpha}\left(\mathbb{R}^{n}\right)$ are equipped with the quasi-norms given by

$$
\left\|f \mid \dot{B}_{p q}^{s, \alpha}\left(\mathbb{R}^{n}\right)\right\|:=\left(\sum_{j=-\infty}^{\infty} 2^{j s q}\left\|\left(\varphi_{j}^{\alpha} \widehat{f}\right)^{\vee} \mid L_{p}\left(\mathbb{R}^{n}\right)\right\|^{q}\right)^{1 / q}
$$

and

$$
\left\|f\left|\dot{F}_{p q}^{s, \alpha}\left(\mathbb{R}^{n}\right)\|:=\|\left(\sum_{j=-\infty}^{\infty} 2^{j s q}\left|\left(\varphi_{j}^{\alpha} \widehat{f}\right)^{\vee}(\cdot)\right|^{q}\right)^{1 / q}\right| L_{p}\left(\mathbb{R}^{n}\right)\right\|,
$$

respectively. Here we have extended the definition of $\left(\varphi_{j}^{\alpha}\right)$ given by (5) to all $j \in \mathbb{Z}$ with the minor modification i.e. for $j=0$, we put $\varphi_{0}^{\alpha}(x)=\varphi^{\alpha}(x)-$ $\varphi^{\alpha}\left(2^{\alpha} x\right)$. Denoting by $\dot{A}_{p q}^{s, \alpha}\left(\mathbb{R}^{n}\right)$ one of the spaces $\dot{B}_{p q}^{s, \alpha}\left(\mathbb{R}^{n}\right)$ or $\dot{F}_{p q}^{s, \alpha}\left(\mathbb{R}^{n}\right)$, we may state the next result.

Proposition 2.3. Let $0<p, q \leq \infty$ with $p<\infty$ in the $F$-case and $s>\sigma_{p}$. Moreover let $\alpha$ be an anisotropy according to (2). Then

$$
\left\|f\left|A_{p q}^{s, \alpha}\left(\mathbb{R}^{n}\right)\|\sim\| f\right| L_{p}\left(\mathbb{R}^{n}\right)\right\|+\left\|f \mid \dot{A}_{p q}^{s, \alpha}\left(\mathbb{R}^{n}\right)\right\|
$$

holds for all $f \in A_{p q}^{s, \alpha}\left(\mathbb{R}^{n}\right)$.

We will also need a "continuous" version of the above proposition replacing the homogeneous quasi-norm on the right-hand side of (12) by its integral counterpart. Note that the Besov space case can be found in $[8$, Theorem $3.3]$.

Theorem 2.4. Let $0<p, q \leq \infty, s>\sigma_{p}$ and let $\alpha$ be an anisotropy according to (2). Moreover, we put $\rho^{\alpha}(t \xi)=\varphi^{\alpha}\left(t^{\alpha} \xi\right)-\varphi^{\alpha}\left((2 t)^{\alpha} \xi\right)$, where $t>0$ and $\varphi^{\alpha}$ as in (4). Then

(i)

$$
\left\|f \mid L_{p}\left(\mathbb{R}^{n}\right)\right\|+\left(\int_{0}^{\infty} t^{-s q}\left\|\left(\rho^{\alpha}(t \cdot) \widehat{f}\right)^{\vee} \mid L_{p}\left(\mathbb{R}^{n}\right)\right\|^{q} \frac{\mathrm{d} t}{t}\right)^{1 / q}
$$

(modification for $q=\infty$ ) is an equivalent quasi-norm in $B_{p q}^{s, \alpha}\left(\mathbb{R}^{n}\right)$. 
(ii)

$$
\begin{aligned}
& \left\|f\left|L_{p}\left(\mathbb{R}^{n}\right)\|+\|\left(\int_{0}^{\infty} t^{-s q}\left|\left(\rho^{\alpha}(t \cdot) \widehat{f}\right)^{\vee}(\cdot)\right|^{q} \frac{\mathrm{d} t}{t}\right)^{1 / q}\right| L_{p}\left(\mathbb{R}^{n}\right)\right\| \\
& \text { (modification for } q=\infty \text { ) is an equivalent quasi-norm in } F_{p q}^{s, \alpha}\left(\mathbb{R}^{n}\right) .
\end{aligned}
$$

Both Proposition 2.3 and Theorem 2.4 can be proved in the same way as in [10, Section 2.3.3]. This will be omitted here.

\subsection{Classical atomic decompositions in anisotropic function spaces.} As a preparation, we shall recall some basic notations of atomic decompositions in the anisotropic setting. If $\nu \in \mathbb{N}_{0}$ and $m=\left(m_{1}, \ldots, m_{n}\right) \in \mathbb{Z}^{n}$, we denote by $Q_{\nu m}^{\alpha}$ the rectangle in $\mathbb{R}^{n}$ with sides parallel to the axes of coordinates, centered at $2^{-\nu \alpha} m=\left(2^{-\nu \alpha_{1}} m_{1}, \ldots, 2^{-\nu \alpha_{n}} m_{n}\right)$ and with side lengths $2^{-(\nu-1) \alpha_{1}}, \ldots, 2^{-(\nu-1) \alpha_{n}}$. In particular, $Q_{0 m}^{\alpha}$ are rectangles of side lengths $2^{\alpha_{1}}, \ldots, 2^{\alpha_{n}}$ centered at $m \in \mathbb{Z}^{n}$. If $Q$ is a rectangle in $\mathbb{R}^{n}$ and $d>0$, then $d Q$ is the rectangle in $\mathbb{R}^{n}$ concentric with $Q$ and with side length $d$ times the side length of $Q$.

We are now in a position to introduce the respective building blocks.

Definition 2.5. Let $\alpha$ be an anisotropy according to (2). Let $s \in \mathbb{R}, 0<$ $p \leq \infty, K, L \geq 0$ and $d>1$. A continuous function $a: \mathbb{R}^{n} \rightarrow \mathbb{C}$ with all derivatives $\mathrm{D}^{\gamma} a$ for $\alpha \gamma \leq K$ is said to be an anisotropic $(s, p)_{K, L^{-}}$atom if

(i) supp $a \subset d Q_{\nu m}^{\alpha} \quad$ for some $\quad \nu \in \mathbb{N}_{0}, m \in \mathbb{Z}^{n}$,

(ii) $\left|\mathrm{D}^{\gamma} a(x)\right| \leq 2^{-\nu\left(s-\frac{n}{p}-\gamma \alpha\right)}$ for $\alpha \gamma \leq K, \quad x \in \mathbb{R}^{n}$,

(iii) $\int_{\mathbb{R}^{n}} x^{\beta} a(x) \mathrm{d} x=0 \quad$ for $\quad \beta \in \mathbb{N}_{0}^{n} \quad$ with $\beta \alpha<L$.

If conditions (i) and (ii) are satisfied for $\nu=0$, then $a$ is called an anisotropic $1_{K}$-atom.

Remark 2.6. In the sequel, we will write $a_{\nu m}^{\alpha}$ instead of $a$, to indicate the

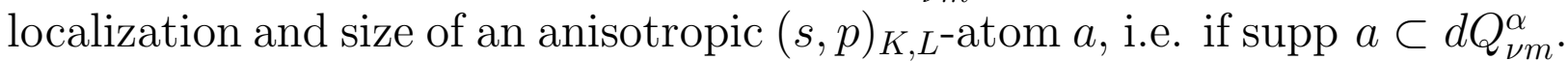
If $L=0$, then (iii) simply means that there are no moment conditions. In this case, we shorten the notation by writing $(s, p)_{K^{-}}$atom instead of $(s, p)_{K, 0^{-}}$ atom.

The main advantage of the atomic decomposition approach is that we can often reduce a problem given in $A_{p q}^{s, \alpha}\left(\mathbb{R}^{n}\right)$ to the corresponding sequence space. We shall resrict ourselves to the case $A=B$ and thus define the Besov sequence spaces. 
Definition 2.7. Let $0<p, q \leq \infty$ and put $\lambda=\left\{\lambda_{\nu m} \in \mathbb{C}: \nu \in \mathbb{N}_{0}, m \in \mathbb{Z}^{n}\right\}$. The Besov sequence space $b_{p q}$ is defined as the set

$$
b_{p q}=\left\{\lambda: \quad\left\|\lambda \mid b_{p q}\right\|:=\left(\sum_{\nu=0}^{\infty}\left(\sum_{m \in \mathbb{Z}^{n}}\left|\lambda_{\nu m}\right|^{p}\right)^{q / p}\right)^{1 / p}<\infty\right\}
$$

with the usual modification if either $p=\infty$ or $q=\infty$. In what follows, we shall abbreviate $b_{p p}$ to $b_{p}$.

In the sequel to shorten the notation we utilize the following abbreviation:

$$
\sigma_{p}=n\left(\frac{1}{p}-1\right)_{+} .
$$

Below we formulate the atomic decomposition characterization of anisotropic Besov spaces $B_{p q}^{s, \alpha}\left(\mathbb{R}^{n}\right)$, following essentially [3, Theorem 3.3].

Theorem 2.8. Let $0<p, q \leq \infty, s \in \mathbb{R}$ and $\alpha$ be an anisotropy according to (2). Let $K, L \geq 0$ with

$$
K \geq\left\{\begin{array}{lll}
0 & \text { for } & s<0 \\
s+\alpha_{n} & \text { for } & s \geq 0
\end{array}\right.
$$

and $L>\sigma_{p}-s$ be fixed.

A tempered distribution $f \in \mathcal{S}^{\prime}\left(\mathbb{R}^{n}\right)$ belongs to $B_{p q}^{s, \alpha}\left(\mathbb{R}^{n}\right)$ if, and only if, it can be written as

$$
f=\sum_{\nu=0}^{\infty} \sum_{m \in \mathbb{Z}^{n}} \lambda_{\nu m} a_{\nu m}^{\alpha}, \quad \text { converging in } \mathcal{S}^{\prime}\left(\mathbb{R}^{n}\right),
$$

where $a_{\nu m}^{\alpha}$ are anisotropic $1_{K}$-atoms $(\nu=0)$ or $(s, p)_{K, L^{-a t o m s}}(\nu \in \mathbb{N})$ and $\lambda \in b_{p q}$. Furthermore

$$
\inf \left\|\lambda \mid b_{p q}\right\|
$$

where the infimum is taken over all admissible representations (17), is an equivalent quasi-norm in $B_{p q}^{s, \alpha}\left(\mathbb{R}^{n}\right)$.

As an application of the above smooth atomic decomposition theorem we obtain the next result. For $K \in \mathbb{N}$ and $\alpha$ an anisotropy we denote by 
$C^{K, \alpha}\left(\mathbb{R}^{n}\right)$ the set of all functions $f \in C\left(\mathbb{R}^{n}\right)$ such that $\mathrm{D}^{\beta} f \in C\left(\mathbb{R}^{n}\right)$ for $\beta \in \mathbb{N}_{0}^{n}$ with $\beta \alpha \leq K$, equipped with the norm given by

$$
\left\|f\left|C^{K, \alpha}\left(\mathbb{R}^{n}\right)\left\|:=\sum_{\beta \alpha \leq K}\right\| \mathrm{D}^{\beta} f\right| L_{\infty}\left(\mathbb{R}^{n}\right)\right\| .
$$

Proposition 2.9. Let $0<p, q \leq \infty, s>\sigma_{p}$ and let $\alpha$ be an anisotropy according to (2). Let $K \in \mathbb{N}$ with $K \geq s+\alpha_{n}$. Then there exists a positive constant $c$ such that

$$
\left\|g f\left|B_{p q}^{s, \alpha}\left(\mathbb{R}^{n}\right)\|\leq c\| g\right| C^{K, \alpha}\left(\mathbb{R}^{n}\right)\right\|\left\|f \mid B_{p q}^{s, \alpha}\left(\mathbb{R}^{n}\right)\right\|,
$$

holds for all $f \in B_{p q}^{s, \alpha}\left(\mathbb{R}^{n}\right)$ and all $g \in C^{K, \alpha}\left(\mathbb{R}^{n}\right)$.

Proof: Let $f \in B_{p q}^{s, \alpha}\left(\mathbb{R}^{n}\right)$ and consider an optimal smooth atomic decomposition

$$
f=\sum_{\nu=0}^{\infty} \sum_{m \in \mathbb{Z}^{n}} \lambda_{\nu m} a_{\nu m}^{\alpha} \quad \text { with } \quad\left\|f\left|B_{p q}^{s, \alpha}\left(\mathbb{R}^{n}\right)\|\sim\| \lambda\right| b_{p q}\right\|,
$$

where $a_{\nu m}^{\alpha}$ are anisotropic $1_{K}$-atoms $(\nu=0)$ or $(s, p)_{K}$-atoms $(\nu \in \mathbb{N})$ and $\lambda=\left(\lambda_{\nu m}\right)_{\nu \in \mathbb{N}, m \in \mathbb{Z}^{n}} \in b_{p q}$. Then, for $g \in C^{K, \alpha}\left(\mathbb{R}^{n}\right)$,

$$
g f=\sum_{\nu=0}^{\infty} \sum_{m \in \mathbb{Z}^{n}} \lambda_{\nu m}\left(g a_{\nu m}^{\alpha}\right)
$$

Note that

$$
\operatorname{supp} g a_{\nu m}^{\alpha} \subset \operatorname{supp} a_{\nu m}^{\alpha} \subset d Q_{\nu m}^{\alpha}
$$

and

$$
\begin{aligned}
\left|\mathrm{D}^{\gamma}\left(g a_{\nu m}^{\alpha}\right)(x)\right| & \leq \sum_{\beta \leq \gamma}\left(\begin{array}{l}
\gamma \\
\beta
\end{array}\right)\left|\mathrm{D}^{\beta} a_{\nu m}^{\alpha}(x)\right|\left|\mathrm{D}^{\gamma-\beta} g(x)\right| \\
& \leq c(\alpha, K)\left\|g \mid C^{K, \alpha}\left(\mathbb{R}^{n}\right)\right\| 2^{-\nu\left(s-\frac{n}{p}-\beta \alpha\right)}
\end{aligned}
$$

for $\beta$ with $\beta \alpha \leq K$. Assuming $g \neq 0$, otherwise (19) is trivially satisfied, we can rewrite $(20)$ as

$$
g f=\sum_{\nu=0}^{\infty} \sum_{m \in \mathbb{Z}^{n}} \sigma_{\nu m} b_{\nu m}
$$

with $\sigma_{\nu m}=c(\alpha, K) \lambda_{\nu m}\left\|g \mid C^{K, \alpha}\left(\mathbb{R}^{n}\right)\right\|$ and $b_{\nu m}(x):=g(x) a_{\nu m}^{\alpha}(x)\left(c(\alpha, K) \lambda_{\nu m}\right.$ $\left.\left\|g \mid C^{K, \alpha}\left(\mathbb{R}^{n}\right)\right\|\right)^{-1}$ being anisotropic $(s, p)_{K^{-a t o m s}}$. Then, by the smooth 
atomic decomposition theorem, it follows that $g f \in B_{p q}^{s, \alpha}\left(\mathbb{R}^{n}\right)$ and, moreover,

$$
\begin{aligned}
\left\|g f \mid B_{p q}^{s, \alpha}\left(\mathbb{R}^{n}\right)\right\| & \leq c_{1}\left\|\sigma \mid b_{p q}\right\| \\
& \leq c_{2}\left\|g\left|C^{K, \alpha}\left(\mathbb{R}^{n}\right)\|\| \lambda\right| b_{p q}\right\| \\
& \leq c_{3}\left\|g\left|C^{K, \alpha}\left(\mathbb{R}^{n}\right)\|\| f\right| B_{p q}^{s, \alpha}\left(\mathbb{R}^{n}\right)\right\|,
\end{aligned}
$$

with constants independent of $f$ and $g$.

\section{Homogeneity property for anisotropic function spaces}

The homogeneity property presented below is based on the Fubini property defined as follows.

Definition 3.1. Let $0<p, q \leq \infty, s>\sigma_{p}$ and let $\alpha$ be an anisotropy according to (2). Then $B_{p q}^{s, \alpha}\left(\mathbb{R}^{n}\right)$ is said to have the Fubini property if

$$
\sum_{r=1}^{n}\|\| f\left(x_{1}, \ldots, x_{r-1}, \cdot, x_{r+1}, \ldots, x_{n}\right)\left|B_{p q}^{s_{r}}(\mathbb{R})\left\|\mid L_{p}\left(\mathbb{R}^{n-1}\right)\right\|\right.
$$

is an equivalent quasi-norm in $B_{p q}^{s, \alpha}\left(\mathbb{R}^{n}\right)$.

Note that the inner quasi-norm in (22) is taken only with respect to the variable $x_{r}$ and $s_{r}=s / \alpha_{r}$.

Theorem 3.2. Let $0<p, q \leq \infty, s>\sigma_{p}$ and let $\alpha$ be an anisotropy according to (2). Then the spaces $B_{p q}^{s, \alpha}\left(\mathbb{R}^{n}\right)$ have the Fubini property if, and only if, $p=q$.

For the proof and more details, we refer the reader to [2]. As we will see below, the Fubini property will play a central role in the proof of the homogeneity property for anisotropic Besov spaces $B_{p}^{s, \alpha}\left(\mathbb{R}^{n}\right)$. The following proposition is a simple consequence of recent results on the homogeneity property in isotropic function spaces on domains due to A. Caetano et al. [1].

Proposition 3.3. Let $0<p, q \leq \infty$ and $s>\sigma_{p}$. Furthermore, let $f \in$ $B_{p q}^{s}\left(\mathbb{R}^{n}\right)$ be such that supp $f \subset\left\{y \in \mathbb{R}^{n}:|y| \leq \lambda\right\}$ for some $0<\lambda<1$. Then

$$
\left\|f(\lambda \cdot)\left|B_{p q}^{s}\left(\mathbb{R}^{n}\right)\left\|\sim \lambda^{s-n / p}\right\| f\right| B_{p q}^{s}\left(\mathbb{R}^{n}\right)\right\|,
$$

where the equivalence constants are independent of $\lambda$. 
For a complete treatment of homogeneity property for isotropic Besov and Triebel-Lizorkin spaces on domains, the reader may consult a recent work of A. Caetano et al. [1]. The next result describes the homogeneity property in special anisotropic Besov spaces, when $p=q$. Let us briefly comment on the anisotropic homogeneity property in Lebesgue spaces $L_{p}\left(\mathbb{R}^{n}\right)$ with $0<p \leq \infty$. A straightforward computation shows that for $\lambda>0$

$$
\left\|f\left(\lambda^{\alpha} \cdot\right)\left|L_{p}\left(\mathbb{R}^{n}\right)\left\|=\lambda^{-\left(\alpha_{1}+\ldots+\alpha_{n}\right) / p}\right\| f\right| L_{p}\left(\mathbb{R}^{n}\right)\right\|=\lambda^{-n / p}\left\|f \mid L_{p}\left(\mathbb{R}^{n}\right)\right\| .
$$

In the sequel, we utilize the following abbreviation

$$
B_{p}^{s, \alpha}\left(\mathbb{R}^{n}\right)=B_{p p}^{s, \alpha}\left(\mathbb{R}^{n}\right), \quad \text { where } 0<p \leq \infty, s \in \mathbb{R}
$$

Proposition 3.4. Let $0<p \leq \infty, s>\sigma_{p}$ and let $\alpha$ be an anisotropy according to (2). Furthermore, let $f \in B_{p}^{s, \alpha}\left(\mathbb{R}^{n}\right)$ be such that supp $f \subset\{y \in$ $\left.\mathbb{R}^{n}:|y|_{\alpha} \leq \lambda\right\}$ for some $0<\lambda<1$. Then

$$
\left\|f\left(\lambda^{\alpha} \cdot\right)\left|B_{p}^{s, \alpha}\left(\mathbb{R}^{n}\right)\left\|\sim \lambda^{s-n / p}\right\| f\right| B_{p}^{s, \alpha}\left(\mathbb{R}^{n}\right)\right\|
$$

where the equivalence constants are independent of $\lambda$.

Proof: The central idea of the proof is the use of the Fubini property for anisotropic Besov spaces $B_{p}^{s, \alpha}\left(\mathbb{R}^{n}\right)$, to obtain an equivalent quasi-norm modeled only on Besov spaces defined on $\mathbb{R}$, which are isotropic. For these spaces we shall employ the homogeneity property of isotropic Besov spaces as described in Proposition 3.3. Assume that $f \in B_{p}^{s, \alpha}\left(\mathbb{R}^{n}\right)$ with supp $f \subset\{y \in$ $\left.\mathbb{R}^{n}:|y|_{\alpha} \leq \lambda\right\}$. Recall that by virtue of Theorem 3.2 we have

$$
\left\|f \left|B _ { p } ^ { s , \alpha } ( \mathbb { R } ^ { n } ) \| \sim \sum _ { r = 1 } ^ { n } \| \left\|f ( x _ { 1 } , \ldots , x _ { r - 1 } , \cdot , x _ { r + 1 } , \ldots , x _ { n } ) \left|B_{p}^{s_{r}}(\mathbb{R})\left\|\mid L_{p}\left(\mathbb{R}^{n-1}\right)\right\|\right.\right.\right.\right.
$$

It may be worth reminding the reader that by (9) we have that $s=\alpha_{r} s_{r}$ for $r=1, \ldots, n$. Applying (26) to $f\left(\lambda^{\alpha} \cdot\right)$, using (23) and (24) results in 


$$
\begin{aligned}
& \left\|f\left(\lambda^{\alpha} \cdot\right) \mid B_{p}^{s, \alpha}\left(\mathbb{R}^{n}\right)\right\| \\
& \sim \sum_{r=1}^{n}\|\| f\left(\lambda^{\alpha_{1}} x_{1}, \ldots, \lambda^{\alpha_{r-1}} x_{r-1}, \lambda^{\alpha_{r}}, \lambda^{\alpha_{r+1}} x_{r+1}, \ldots, \lambda^{\alpha_{n}} x_{n}\right)\left|B_{p}^{s_{r}}(\mathbb{R})\left\|\mid L_{p}\left(\mathbb{R}^{n-1}\right)\right\|\right. \\
& \sim \sum_{r=1}^{n}\left\|\left(\lambda^{\alpha_{r}}\right)^{s_{r}-\frac{1}{p}}\right\| f\left(\lambda^{\alpha_{1}} x_{1}, \ldots, \lambda^{\alpha_{r-1}} x_{r-1}, \cdot, \lambda^{\alpha_{r+1}} x_{r+1}, \ldots, \lambda^{\alpha_{n}} x_{n}\right)\left|B_{p}^{s_{r}}(\mathbb{R})\left\|\mid L_{p}\left(\mathbb{R}^{n-1}\right)\right\|\right. \\
& \sim \lambda^{s-\frac{\alpha_{r}}{p}} \lambda^{-\frac{\alpha_{1}+\ldots+\alpha_{r-1}+\alpha_{r+1}+\ldots+\alpha_{n}}{p}} \sum_{r=1}^{n}\|\| f(\cdot)\left|B_{p}^{s_{r}}(\mathbb{R})\left\|\mid L_{p}\left(\mathbb{R}^{n-1}\right)\right\|\right. \\
& =\lambda^{s-n / p} \sum_{r=1}^{n}\|\| f(\cdot)\left|B_{p}^{s_{r}}(\mathbb{R})\left\|\left|L_{p}\left(\mathbb{R}^{n-1}\right)\left\|\sim \lambda^{s-n / p}\right\| f(\cdot)\right| B_{p}^{s, \alpha}\left(\mathbb{R}^{n}\right)\right\|,\right.
\end{aligned}
$$

which finishes the proof.

Next, we make full use of Theorem 2.4 to get the following assertion.

Proposition 3.5. Let $f \in A_{p q}^{s, \alpha}\left(\mathbb{R}^{n}\right)$ with $s>\sigma_{p}\left(s>\sigma_{p q}\right.$ in the F-case). Then

$$
\left\|f\left(\lambda^{\alpha} \cdot\right)\left|A_{p q}^{s, \alpha}\left(\mathbb{R}^{n}\right)\left\|\sim \lambda^{s-n / p}\right\| f(\cdot)\right| \dot{A}_{p q}^{s, \alpha}\left(\mathbb{R}^{n}\right)\right\|+\lambda^{-n / p}\left\|f \mid L_{p}\left(\mathbb{R}^{n}\right)\right\|
$$

holds for $\lambda>0$. The underlying equivalence constants are independent of $\lambda$.

Proof: Taking into account the equivalent quasi-norm in $A_{p q}^{s, \alpha}\left(\mathbb{R}^{n}\right)$ given by (12) with $f\left(\lambda^{\alpha} \cdot\right)$ in place of $f(\cdot)$ yields

$$
\begin{aligned}
\left\|f\left(\lambda^{\alpha} \cdot\right) \mid A_{p q}^{s, \alpha}\left(\mathbb{R}^{n}\right)\right\| & \sim\left\|f\left(\lambda^{\alpha} \cdot\right)\left|L_{p}\left(\mathbb{R}^{n}\right)\|+\| f\left(\lambda^{\alpha} \cdot\right)\right| \dot{A}_{p q}^{s, \alpha}\left(\mathbb{R}^{n}\right)\right\| \\
& \sim \lambda^{-n / p}\left\|f\left|L_{p}\left(\mathbb{R}^{n}\right)\|+\| f\left(\lambda^{\alpha} \cdot\right)\right| \dot{A}_{p q}^{s, \alpha}\left(\mathbb{R}^{n}\right)\right\| .
\end{aligned}
$$

The last equivalence follows from (24). Recall that $\rho^{\alpha}(t \xi)=\varphi\left(t^{\alpha} \xi\right)-$ $\varphi\left((2 t)^{\alpha} \xi\right)$. More precisely,

$$
\rho^{\alpha}(t \xi)=\varphi\left(t^{\alpha_{1}} \xi_{1}, \ldots, t^{\alpha_{n}} \xi_{n}\right)-\varphi\left((2 t)^{\alpha_{1}} \xi_{1}, \ldots,(2 t)^{\alpha_{n}} \xi_{n}\right) .
$$

Therefore, a chain of standard substitutions gives

$$
\begin{aligned}
\left(\rho^{\alpha}(t \cdot) \mathcal{F}\left(f\left(\lambda^{\alpha} \cdot\right)\right)(\cdot)\right)^{\vee}(x) & =\left(\rho^{\alpha}(t \cdot) \lambda^{-n} \mathcal{F}(f(\cdot))\left(\lambda^{-\alpha} \cdot\right)\right)^{\vee}(x) \\
& =\left(\rho^{\alpha}((\lambda t) \cdot) \mathcal{F}(f(\cdot))(\cdot)\right)^{\vee}\left(\lambda^{\alpha} x\right) .
\end{aligned}
$$


To establish the proof, we consider the integral part of the equivalent quasinorms given by (13) and (14). We state it here for $A=B$. Then, we obtain

$$
\begin{aligned}
\left\|f\left(\lambda^{\alpha} \cdot\right) \mid \dot{B}_{p q}^{s, \alpha}\left(\mathbb{R}^{n}\right)\right\| & \sim\left(\int_{0}^{\infty} t^{-s q}\left\|\left(\rho^{\alpha}(t \cdot) \mathcal{F}\left(f\left(\lambda^{\alpha} \cdot\right)\right)(\cdot)\right)^{\vee} \mid L_{p}\left(\mathbb{R}^{n}\right)\right\|^{q} \frac{\mathrm{d} t}{t}\right)^{1 / q} \\
& =\left(\int_{0}^{\infty} t^{-s q}\left\|\left(\rho^{\alpha}((\lambda t) \cdot) \widehat{f}(\cdot)\right)^{\vee}\left(\lambda^{\alpha} \cdot\right) \mid L_{p}\left(\mathbb{R}^{n}\right)\right\|^{q} \frac{\mathrm{d} t}{t}\right)^{1 / q} \\
& =\left(\int_{0}^{\infty} \frac{(\lambda t)^{-s q}}{\lambda^{-s q}}\left\|\left(\rho^{\alpha}((\lambda t) \cdot) \widehat{f}(\cdot)\right)^{\vee}\left(\lambda^{\alpha} \cdot\right) \mid L_{p}\left(\mathbb{R}^{n}\right)\right\|^{q} \frac{\mathrm{d} t}{t}\right)^{1 / q} \\
& \sim \lambda^{s-n / p}\left(\int_{0}^{\infty} t^{-s q}\left\|\left(\rho^{\alpha}(t \cdot) \widehat{f}(\cdot)\right)^{\vee}(\cdot) \mid L_{p}\left(\mathbb{R}^{n}\right)\right\|^{q} \frac{\mathrm{d} t}{t}\right)^{1 / q} \\
& \sim \lambda^{s-n / p}\left\|f \mid \dot{B}_{p q}^{s, \alpha}\left(\mathbb{R}^{n}\right)\right\|,
\end{aligned}
$$

which finishes the proof for the $B$-case. The proof of the $F$-case is analogous.

\section{Anisotropic non-smooth atoms}

Definition 4.1. Let $c \geq 1,0<p \leq \infty$ and $\sigma_{p}<s<\sigma<\infty$, where $\sigma_{p}$ is given by (15). Then $a_{\nu m}^{\alpha} \in B_{p}^{\sigma, \alpha}\left(\mathbb{R}^{n}\right)$ is called an anisotropic $(s, p)^{\sigma}$-atom provided that

$$
\operatorname{supp} a_{\nu m}^{\alpha} \subset c Q_{\nu m}^{\alpha} \quad \text { where } \nu \in \mathbb{N}_{0}, m \in \mathbb{Z}^{n}
$$

and

$$
\left\|a_{\nu m}^{\alpha} \mid B_{p}^{\sigma, \alpha}\left(\mathbb{R}^{n}\right)\right\| \leq 2^{\nu(\sigma-s)} .
$$

The next proposition summarizes the basic properties of the just introduced anisotropic non-smooth atoms. In its first part we compare these atoms with the classical atoms described in Definition 2.5.

Proposition 4.2. Let $c \geq 1, \nu \in \mathbb{N}_{0}$ and $m \in \mathbb{Z}^{n}$. Moreover let $0<p \leq \infty$ and $\sigma_{p}<s<\sigma$.

(i) Let $\sigma+\alpha_{n} \leq K \in \mathbb{N}$. Then any anisotropic $(s, p)_{K}$-atom $a_{\nu m}^{\alpha}$ according to Definition 2.5 is an anisotropic $(s, p)^{\sigma}$-atom as introduced in Definition 4.1.

(ii) Let $a_{\nu m}^{\alpha}$ be an anisotropic $(s, p)^{\sigma}$-atom. Then

$$
\left\|a_{\nu m}^{\alpha} \mid B_{p}^{s, \alpha}\left(\mathbb{R}^{n}\right)\right\| \leq 1
$$


In particular, for $p \geq 1$ we obtain

$$
\left\|a_{\nu m}^{\alpha} \mid L_{p}\left(\mathbb{R}^{n}\right)\right\| \leq 2^{-\nu s}
$$

Proof: Let us start by recalling the needed homogeneity property. Taking $\lambda=2^{-\nu}, \nu \in \mathbb{N}$ in Proposition 3.4 we obtain for $g \in B_{p}^{s, \alpha}\left(\mathbb{R}^{n}\right)$ with supp $g \subset$ $\left\{y \in \mathbb{R}^{n}:|y|_{\alpha} \leq 1\right\}$ that

$$
\left\|g\left|B_{p}^{s, \alpha}\left(\mathbb{R}^{n}\right)\left\|\sim 2^{-\nu(s-n / p)}\right\| g\left(2^{\nu \alpha} \cdot\right)\right| B_{p}^{s, \alpha}\left(\mathbb{R}^{n}\right)\right\| .
$$

To establish (i) let us assume that $a_{\nu m}^{\alpha}$ is an anisotropic $(s, p)_{K}$-atom with $K>\sigma>s$. We can write

$$
a_{\nu m}^{\alpha}(x)=2^{\nu(\sigma-s)} b_{\nu m}^{\alpha}(x),
$$

where

$$
b_{\nu m}^{\alpha}(x):=2^{\nu(s-\sigma)} a_{\nu m}^{\alpha}(x), \quad x \in \mathbb{R}^{n}, \nu \in \mathbb{N}_{0}, m \in \mathbb{Z}^{n} .
$$

Note that, for each $\nu \in \mathbb{N}_{0}$ and $m \in \mathbb{Z}^{n}$, we have

$$
\operatorname{supp} b_{\nu m}^{\alpha}=\operatorname{supp} a_{\nu m}^{\alpha} \subset c Q_{\nu m}^{\alpha}
$$

and

$$
\left|\mathrm{D}^{\gamma} b_{\nu m}^{\alpha}(x)\right| \leq 2^{-\nu\left(\sigma-\frac{n}{p}-\gamma \alpha\right)} \quad \text { for } \quad \gamma \alpha \leq K,
$$

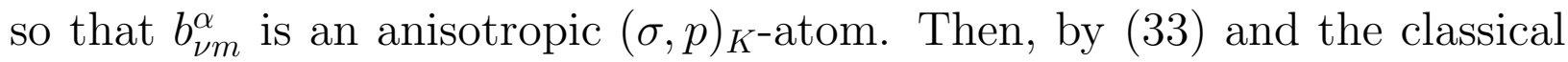
atomic decomposition theorem it follows

$$
a_{\nu m}^{\alpha} \in B_{p}^{\sigma, \alpha}\left(\mathbb{R}^{n}\right) \quad \text { and } \quad\left\|a_{\nu m}^{\alpha} \mid B_{p}^{\sigma, \alpha}\left(\mathbb{R}^{n}\right)\right\| \lesssim 2^{\nu(\sigma-s)}
$$

and hence $a_{\nu m}^{\alpha}$ are anisotropic $(s, p)^{\sigma}$-atoms.

We now prove (ii). We may assume $m=0$ and we put $a_{\nu}^{\alpha} \equiv a_{\nu 0}^{\alpha}$. Applying (32) to $g(x)=a_{\nu}^{\alpha}\left(2^{-\nu \alpha} x\right)$ and using the elementary embedding $B_{p}^{\sigma, \alpha}\left(\mathbb{R}^{n}\right) \hookrightarrow$ $B_{p}^{s, \alpha}\left(\mathbb{R}^{n}\right)$, we obtain for $\nu \in \mathbb{N}_{0}$

$$
\begin{aligned}
\left\|a_{\nu}^{\alpha} \mid B_{p}^{s, \alpha}\left(\mathbb{R}^{n}\right)\right\| & \sim 2^{\nu(s-n / p)}\left\|a_{\nu}^{\alpha}\left(2^{-\nu \alpha} \cdot\right) \mid B_{p}^{s, \alpha}\left(\mathbb{R}^{n}\right)\right\| \\
& \lesssim 2^{\nu(s-n / p)}\left\|a_{\nu}^{\alpha}\left(2^{-\nu \alpha} \cdot\right) \mid B_{p}^{\sigma, \alpha}\left(\mathbb{R}^{n}\right)\right\| \\
& \lesssim 2^{-\nu(\sigma-s)}\left\|a_{\nu}^{\alpha} \mid B_{p}^{\sigma, \alpha}\left(\mathbb{R}^{n}\right)\right\| \\
& \lesssim 1 .
\end{aligned}
$$

Let $r \in(1, \infty)$ be such that $r>p$ and $s-n / p \geq-n / r$. Then it holds

$$
B_{p}^{s, \alpha}\left(\mathbb{R}^{n}\right)=F_{p, p}^{s, \alpha}\left(\mathbb{R}^{n}\right) \hookrightarrow F_{r, 2}^{0, \alpha}\left(\mathbb{R}^{n}\right)=L_{r}\left(\mathbb{R}^{n}\right) .
$$


Using the Hölder inequality combined with the homogeneity property (32) we obtain for $\nu \in \mathbb{N}_{0}$

$$
\begin{aligned}
\left\|a_{\nu}^{\alpha} \mid L_{p}\left(\mathbb{R}^{n}\right)\right\| & =2^{-\nu n / p}\left\|a_{\nu}^{\alpha}\left(2^{-\nu \alpha} \cdot\right) \mid L_{p}\left(\mathbb{R}^{n}\right)\right\| \\
& \lesssim 2^{-\nu n / p}\left\|a_{\nu}^{\alpha}\left(2^{-\nu \alpha} \cdot\right) \mid L_{r}\left(\mathbb{R}^{n}\right)\right\| \\
& \lesssim 2^{-\nu n / p}\left\|a_{\nu}^{\alpha}\left(2^{-\nu \alpha} \cdot\right) \mid B_{p}^{s, \alpha}\left(\mathbb{R}^{n}\right)\right\| \\
& \lesssim 2^{-\nu n / p} 2^{-\nu(s-n / p)}\left\|a_{\nu}^{\alpha} \mid B_{p}^{s, \alpha}\left(\mathbb{R}^{n}\right)\right\| \\
& \lesssim 2^{-\nu s} .
\end{aligned}
$$

The main result in this section is the following atomic decomposition theorem of type (17) and (18) based on the atoms introduced in Definition 4.1.

Theorem 4.3. Let $0<p \leq \infty, \alpha$ be an anisotropy and $\sigma_{p}<s<\sigma$. Then $B_{p}^{s, \alpha}\left(\mathbb{R}^{n}\right)$ is the collection of all $f \in L_{1}^{\text {loc }}\left(\mathbb{R}^{n}\right) \cap \mathcal{S}^{\prime}\left(\mathbb{R}^{n}\right)$ which can be represented as

$$
f=\sum_{\nu=0}^{\infty} \sum_{m \in \mathbb{Z}^{n}} \lambda_{\nu m} a_{\nu m}^{\alpha}
$$

where $a_{\nu m}^{\alpha}$ for fixed $c \geq 1$ are anisotropic $(s, p)^{\sigma}$-atoms according to Definition 4.1 and $\lambda \in b_{p}$. The series on the right-hand side of (34) converges unconditionally in $\mathcal{S}^{\prime}\left(\mathbb{R}^{n}\right)$ and if $p<\infty$, absolutely in some $L_{r}\left(\mathbb{R}^{n}\right)$ with $1<r<\infty$. Furthermore,

$$
\inf \left\|\lambda \mid b_{p}\right\|
$$

where the infimum is taken over all admissible representations (34), is an equivalent quasi-norm in $B_{p}^{s, \alpha}\left(\mathbb{R}^{n}\right)$.

Proof: Our method will be an adaptation of the reasoning used in [13, Section $2.2]$, but we have to examine very carefully the influence of the anisotropy. Step 1. We start our proof by justifying the convergence of the series on the right-hand side of (34) in some $L_{r}\left(\mathbb{R}^{n}\right)$ with $1<r<\infty$. Assume first that $p>1$. Then, by Proposition 4.2 combined with the support property (28), we obtain

$$
\left\|f\left|L_{p}\left(\mathbb{R}^{n}\right)\left\|\lesssim \sum_{\nu=0}^{\infty} 2^{-\nu s}\left(\sum_{m \in \mathbb{Z}^{n}}\left|\lambda_{\nu m}\right|^{p}\right)^{1 / p} \lesssim\right\| \lambda\right| b_{p}\right\| .
$$


Consequently, the series (34) converges absolutely in $L_{r}\left(\mathbb{R}^{n}\right)$ with $r=p$. In order to clarify the convergence of the series (34) in some $L_{r}\left(\mathbb{R}^{n}\right)$ in the case $p \leq 1$, we utilize the Sobolev embedding

$$
B_{p}^{s, \alpha}\left(\mathbb{R}^{n}\right) \hookrightarrow B_{r}^{t, \alpha}\left(\mathbb{R}^{n}\right) \quad \text { with } \quad s-n / p=t-n / r \quad \text { and } \quad p \leq r .
$$

Step 2. By Theorem 2.8 and Proposition 4.2 the only point remaining concerns the proof of the inequality

$$
\left\|f\left|B_{p}^{s, \alpha}\left(\mathbb{R}^{n}\right)\|\leq c\| \lambda\right| b_{p}\right\|
$$

for all decompositions (34). Taking into account that $B_{p}^{s, \alpha}\left(\mathbb{R}^{n}\right)$ with $p \leq 1$ is a $p$-Banach space combined with Proposition 4.2 (ii) yields

$$
\left\|f\left|B_{p}^{s, \alpha}\left(\mathbb{R}^{n}\right)\left\|^{p} \leq \sum_{\nu=0}^{\infty} \sum_{m \in \mathbb{Z}^{n}}\left|\lambda_{\nu m}\right|^{p}\right\| a_{\nu m}^{\alpha}\right| B_{p}^{s, \alpha}\left(\mathbb{R}^{n}\right)\right\|^{p} \lesssim\left\|\lambda \mid b_{p}\right\|^{p} .
$$

Thus, we are left with the task of proving (36) with $p>1$. We adopt throughout the notational convention that the elements of $\mathbb{N}_{0}$ are denoted by $j, k$ and the elements of $\mathbb{Z}^{n}$ are denoted by $m, w$. Moreover $a^{\alpha}, b^{\alpha}, d^{\alpha}$ denote anisotropic atoms, whereas $\lambda, \eta, \nu$ stand for complex numbers of sequences or complex numbers. Let us rewrite (34) as

$$
f=\sum_{k=0}^{\infty} \sum_{m \in \mathbb{Z}^{n}} \lambda_{k, m} a_{k, m}^{\alpha}
$$

Consider an optimal smooth atomic decomposition of $a_{k, m}^{\alpha}\left(2^{-k \alpha} \cdot\right)$ in $B_{p}^{\sigma, \alpha}\left(\mathbb{R}^{n}\right)$ by smooth anisotropic $(\sigma, p)_{K^{-} \text {atoms }} b_{j, w}^{k, m}$ with $\sigma+\alpha_{n} \leq K$. By virtue of $(17)$ we have

$$
a_{k, m}^{\alpha}\left(2^{-k \alpha} x\right)=\sum_{j=0}^{\infty} \sum_{w \in \mathbb{Z}^{n}} \eta_{j, w}^{k, m} b_{j, w}^{k, m}(x), \quad x \in \mathbb{R}^{n}
$$

with

$$
\operatorname{supp} b_{j, w}^{k, m} \subset d Q_{j w}^{\alpha}, \quad\left|\mathrm{D}^{\gamma} b_{j, w}^{k, m}(x)\right| \leq 2^{-j\left(\sigma-\frac{n}{p}-\gamma \alpha\right)}
$$

for $\alpha \gamma \leq K$ and $x \in \mathbb{R}^{n}$. In addition, one gets

$$
\begin{aligned}
& \left(\sum_{j, w}\left|\eta_{j, w}^{k, m}\right|^{p}\right)^{1 / p} \sim\left\|a_{k, m}^{\alpha}\left(2^{-k \alpha} \cdot\right) \mid B_{p}^{\sigma, \alpha}\left(\mathbb{R}^{n}\right)\right\| \\
& \quad \sim 2^{-k(\sigma-n / p)}\left\|a_{k, m}^{\alpha} \mid B_{p}^{\sigma, \alpha}\left(\mathbb{R}^{n}\right)\right\| \lesssim 2^{-k(\sigma-n / p)} 2^{k(\sigma-s)}=2^{-k(s-n / p)} .
\end{aligned}
$$


Consequently,

$$
a_{k, m}^{\alpha}(x)=\sum_{j=0}^{\infty} \sum_{w \in \mathbb{Z}^{n}} \eta_{j, w}^{k, m} b_{j, w}^{k, m}\left(2^{k \alpha} x\right), \quad x \in \mathbb{R}^{n},
$$

where the functions $b_{j, w}^{k, m}\left(2^{k \alpha}\right.$.) have supports in $c Q_{j+k, w}^{\alpha}$. Namely, we have

$$
\begin{aligned}
\operatorname{supp} b_{j, w}^{k, m}\left(2^{k \alpha} \cdot\right) & =\left\{x \in \mathbb{R}^{n}: \quad\left|2^{k \alpha_{i}} x_{i}-2^{-j \alpha_{i}} w_{i}\right| \leq c 2^{-j \alpha_{i}}, i=1, \ldots, n\right\} \\
& =\left\{x \in \mathbb{R}^{n}: \quad\left|x_{i}-2^{-(j+k) \alpha_{i}} w_{i}\right| \leq c 2^{-(j+k) \alpha_{i}}, i=1, \ldots, n\right\} \\
& =c Q_{j+k, w}^{\alpha} .
\end{aligned}
$$

Furthermore, by virtue of (38), we obtain

$$
\begin{aligned}
& \left|\mathrm{D}^{\gamma} b_{j, w}^{k, m}\left(2^{k \alpha} x\right)\right|=2^{k \alpha \gamma}\left|\left(\mathrm{D}^{\gamma} b_{j, w}^{k, m}\right)\left(2^{k \alpha} x\right)\right| \\
& \leq 2^{(j+k) \alpha \gamma} 2^{-j(\sigma-n / p)}=2^{(j+k) \alpha \gamma} 2^{-(j+k)(\sigma-n / p)} 2^{-(j+k)(\sigma-s)} 2^{k(\sigma-n / p)} .
\end{aligned}
$$

Replacing $j+k$ by $j$ yields

$$
a_{k, m}^{\alpha}(x)=2^{k(\sigma-n / p)} \sum_{j \geq k} \sum_{w \in \mathbb{Z}^{n}} \eta_{j-k, w}^{k, m} 2^{-j(\sigma-s)} d_{j, w}^{k, m}(x),
$$

where $d_{j, w}^{k, m}$ are classical anisotropic $(s, p)_{K}$-atoms. Let $(j, w, k)$ with $k \leq j$ denote the set of all $m \in \mathbb{Z}^{n}$ for which the atoms $d_{j, w}^{k, m}$ in (41) do not vanish, that is,

$$
(j, w, k):=\left\{m \in \mathbb{Z}^{n}: c Q_{k, m}^{\alpha} \cap c Q_{j, w}^{\alpha} \neq \emptyset\right\} .
$$

Note that, if there exists an $x=\left(x_{i}\right)_{i=1}^{n} \in c Q_{k, m}^{\alpha} \cap c Q_{j, w}^{\alpha}$ then

$$
\left|2^{-j \alpha_{i}} w_{i}-2^{-k \alpha_{i}} m_{i}\right| \leq\left|2^{-j \alpha_{i}} w_{i}-x_{i}\right|+\left|2^{-k \alpha_{i}} m_{i}-x_{i}\right| \leq c 2^{-j \alpha_{i}-1}+c 2^{-k \alpha_{i}-1} \text {, }
$$

where $i=1, \cdots, n$, and hence, as $k \leq j$,

$$
\left|2^{(k-j) \alpha_{i}} w_{i}-m_{i}\right| \leq c 2^{(k-j) \alpha_{i}-1}+c 2^{-1} \leq c, \quad i=1, \cdots, n,
$$

which means that, for each $i \in\{1, \cdots, n\}$, there are, at most, $2 c$ possible values for $m_{i}$. Therefore, the cardinal number of $(j, w, k)$ is less or equal to $(2 c)^{n}$ (a number independent of $j, w, k$ ). Let

$$
d_{j, w}^{\alpha}(x)=\frac{\sum_{k \leq j} 2^{k(\sigma-n / p)} \sum_{m \in(j, w, k)} \eta_{j-k, w}^{k, m} \lambda_{k, m} d_{j, w}^{k, m}(x)}{\sum_{k \leq j} 2^{k(\sigma-n / p)} \sum_{m \in(j, w, k)}\left|\eta_{j-k, w}^{k, m}\right|\left|\lambda_{k, m}\right|} .
$$


We can assume that, for $m \in(j, w, k), d_{j, w}^{k, m}$ are smooth anisotropic $(s, p)_{K^{-}}$ atoms with supports in $c Q_{k, m}^{\alpha} \cap c Q_{j, w}^{\alpha}$. Thus, by the definition of $d_{j, w}^{\alpha}$, it clearly follows

$$
\operatorname{supp} d_{j, w}^{\alpha} \subset \bigcup_{k \leq j} \bigcup_{m \in(j, w, k)} \operatorname{supp} d_{j, w}^{k, m} \subset c Q_{j, w}^{\alpha}
$$

and

$$
\left|\mathrm{D}^{\gamma} d_{j, w}^{\alpha}(x)\right| \leq 2^{-j\left(s-\frac{n}{p}-\gamma \alpha\right)} \quad \text { for } \quad \gamma \alpha \leq K,
$$

and hence, $d_{j, w}^{\alpha}$ are smooth anisotropic $(s, p)_{K}$-atoms. Thus, we have

$$
f=\sum_{j=0}^{\infty} \sum_{w \in \mathbb{Z}^{n}} v_{j, w} d_{j, w}^{\alpha},
$$

where

$$
v_{j, w}=2^{-j(\sigma-s)} \sum_{k \leq j} 2^{k(\sigma-n / p)} \sum_{m \in(j, w, k)}\left|\eta_{j-k, w}^{k, m}\right|\left|\lambda_{k, m}\right| .
$$

Choosing $0<\varepsilon<\sigma-s$, we get for $p<\infty$

$$
\begin{aligned}
\left|v_{j, w}\right|^{p} & \lesssim \sum_{k \leq j} \sum_{m \in(j, w, k)} 2^{-(j-k)(\sigma-s-\varepsilon) p} 2^{k(\sigma-n / p) p}\left|\eta_{j-k, w}^{k, m}\right|^{p}\left|\lambda_{k, m}\right|^{p} \\
& \leq \sum_{k \leq j} \sum_{m \in(j, w, k)} 2^{k(\sigma-n / p)}\left|\eta_{j-k, w}^{k, m}\right|^{p}\left|\lambda_{k, m}\right|^{p} .
\end{aligned}
$$

Finally, the above estimate combined with (39) gives

$$
\begin{aligned}
\sum_{j=0}^{\infty} \sum_{w \in \mathbb{Z}^{n}}\left|v_{j, w}\right|^{p} & \lesssim \sum_{k=0}^{\infty} \sum_{m \in \mathbb{Z}^{n}}\left|\lambda_{k, m}\right|^{p} \sum_{j \geq k} \sum_{w \in \mathbb{Z}^{n}} 2^{k(\sigma-n / p) p}\left|\eta_{j-k, w}^{k, m}\right|^{p} \\
& \lesssim \sum_{k=0}^{\infty} \sum_{m \in \mathbb{Z}^{n}}\left|\lambda_{k, m}\right|^{p} .
\end{aligned}
$$

Consequently, (42) is a decomposition by smooth atoms and (36) follows from Theorem 2.8 and the last estimate.

As an easy consequence of Proposition 4.2(i) and Theorem 4.3 we obtain the following smooth atomic decomposition. Remark that the smoothness property of the classical anisotropic atoms used below does not depend on the given anisotropy as it is in (16). 
Corollary 4.4. Let $0<p \leq \infty$ and $\alpha$ be an anisotropy according to (2). Moreover let $\sigma_{p}<s<K$. Then $B_{p}^{s, \alpha}\left(\mathbb{R}^{n}\right)$ consists of all $f \in L_{1}^{\text {loc }}\left(\mathbb{R}^{n}\right) \cap \mathcal{S}^{\prime}\left(\mathbb{R}^{n}\right)$ which can be written as

$$
f=\sum_{\nu=0}^{\infty} \sum_{m \in \mathbb{Z}^{n}} \lambda_{\nu m} a_{\nu m}^{\alpha},
$$

where $a_{\nu m}^{\alpha}$ for fixed $c \geq 1$ are anisotropic $(s, p)_{K}$-atoms according to Definition 2.5 and $\lambda \in b_{p}$.

\section{Pointwise multipliers in anisotropic function spaces}

Let $A^{\alpha}\left(\mathbb{R}^{n}\right)$ denote either $B_{p q}^{s, \alpha}\left(\mathbb{R}^{n}\right)$ or $F_{p q}^{s, \alpha}\left(\mathbb{R}^{n}\right)$ according to Definition 2.1 with $0<p, q \leq \infty(p<\infty$ in the $F$-case $)$ and $s>\sigma_{p}$. However, we will be mostly concerned with $A^{\alpha}\left(\mathbb{R}^{n}\right)=B_{p q}^{s, \alpha}\left(\mathbb{R}^{n}\right)$. A locally integrable function $m$ in $\mathbb{R}^{n}$ is called a pointwise multiplier for $A^{\alpha}\left(\mathbb{R}^{n}\right)$ if

$$
f \mapsto m f
$$

generates a bounded map in $A^{\alpha}\left(\mathbb{R}^{n}\right)$. Since $s>\sigma_{p}$, the spaces under consideration are embedded in some $L_{r}\left(\mathbb{R}^{n}\right)$ with $1<r \leq \infty$ and therefore, the expression $m f$ above makes sense as a product of functions. The collection of all multipliers for $A^{\alpha}\left(\mathbb{R}^{n}\right)$ is denoted by $M\left(A^{\alpha}\left(\mathbb{R}^{n}\right)\right)$. In the sequel, let $\psi$ stand for a non-negative $C^{\infty}$ function with

$$
\operatorname{supp} \psi \subset\left\{y \in \mathbb{R}^{n}:|y|_{\alpha} \leq \sqrt{n}\right\}
$$

and

$$
\sum_{l \in \mathbb{Z}^{n}} \psi(x-l)=1, \quad x \in \mathbb{R}^{n} .
$$

Definition 5.1. Let $0<p, q \leq \infty(p<\infty$ in the $F$-case $), s \in \mathbb{R}$ and let $\alpha$ be an anisotropy according to $(2)$. We define the space $A_{\text {selfs }}^{\alpha}\left(\mathbb{R}^{n}\right)$ to be the set of all $f \in \mathcal{S}^{\prime}\left(\mathbb{R}^{n}\right)$ such that

$$
\left\|f\left|A_{\text {selfs }}^{\alpha}\left(\mathbb{R}^{n}\right)\left\|:=\sup _{j \in \mathbb{N}_{0}, l \in \mathbb{Z}^{n}}\right\| \psi(\cdot-l) f\left(2^{-j \alpha} \cdot\right)\right| A^{\alpha}\left(\mathbb{R}^{n}\right)\right\|
$$

is finite.

Remark 5.2. The isotropic selfsimilar spaces were firstly introduced in [12] and considered again in [13] Section 2.3. A careful look at (45) reveals that these space are closely connected with pointwise multipliers. We also mention 
its forerunner, the so-called uniform spaces, which were studied in detail in [5]. Using Proposition 3.5, one can easily show that

$$
A_{\mathrm{selfs}}^{\alpha}\left(\mathbb{R}^{n}\right) \hookrightarrow L_{\infty}\left(\mathbb{R}^{n}\right)
$$

Applying (27) to $f \in A_{\text {selfs }}^{\alpha}\left(\mathbb{R}^{n}\right)$ gives

$$
\begin{aligned}
\| \psi(\cdot-l) & f\left(2^{-j \alpha} \cdot\right) \mid A_{p q}^{s, \alpha}\left(\mathbb{R}^{n}\right) \| \\
& \sim 2^{-j(s-n / p)}\left\|\psi\left(2^{j \alpha} \cdot-l\right) f\left|\dot{A}_{p q}^{s, \alpha}\left(\mathbb{R}^{n}\right)\left\|+2^{j n / p}\right\| \psi\left(2^{j \alpha} \cdot-l\right) f\right| L_{p}\left(\mathbb{R}^{n}\right)\right\|
\end{aligned}
$$

uniformly for all $j \in \mathbb{N}_{0}$ and $l \in \mathbb{Z}^{n}$. Consequently,

$$
2^{j n} \int_{\mathbb{R}^{n}}\left|\psi\left(2^{j \alpha} y-l\right)\right|^{p}|f(y)|^{p} \mathrm{~d} y \leq c\left\|f \mid A_{\mathrm{selfs}}^{\alpha}\left(\mathbb{R}^{n}\right)\right\|^{p}
$$

Thus, the right-hand side of (46) is a uniform bound for $|f(\cdot)|^{p}$ at its (anisotropic) Lebesgue points, which proves the desired embedding, see [6, Corollary p. 13]. The interested reader is referred to [4, Section 3] for further embedding assertions of anisotropic spaces into $L_{\infty}\left(\mathbb{R}^{n}\right)$.

Definition 5.3. Let $0<p \leq \infty$ and $s>\sigma_{p}$. Moreover, let $\alpha$ be an anisotropy according to $(2)$. We define

$$
B_{p, \text { selfs }}^{s+, \alpha}\left(\mathbb{R}^{n}\right):=\bigcup_{\sigma>s} B_{p, \text { selfs }}^{\sigma, \alpha}\left(\mathbb{R}^{n}\right)
$$

Theorem 5.4. Let $0<p \leq \infty$ and $\sigma_{p}<s<\sigma$. Moreover, let $\alpha$ be an anisotropy according to (2).

(i) Then

$$
B_{p, \text { selfs }}^{s+, \alpha}\left(\mathbb{R}^{n}\right) \subset M\left(B_{p}^{s, \alpha}\left(\mathbb{R}^{n}\right)\right) \hookrightarrow B_{p, \text { selfs }}^{s, \alpha}\left(\mathbb{R}^{n}\right)
$$

(ii) Additionally, for $0<p \leq 1$ we get

$$
M\left(B_{p}^{s, \alpha}\left(\mathbb{R}^{n}\right)\right)=B_{p, \text { selfs }}^{s, \alpha}\left(\mathbb{R}^{n}\right) .
$$


Proof: We start by proving the right-hand side embedding in (i). Let $m \in$ $M\left(B_{p}^{s, \alpha}\left(\mathbb{R}^{n}\right)\right)$. Then, using the homogeneity property yields

$$
\begin{aligned}
\| \psi(\cdot-l) & m\left(2^{-j \alpha} \cdot\right) \mid B_{p}^{s, \alpha}\left(\mathbb{R}^{n}\right) \| \\
& \sim 2^{-j\left(s-\frac{n}{p}\right)}\left\|\psi\left(2^{j \alpha} \cdot-l\right) m \mid B_{p}^{s, \alpha}\left(\mathbb{R}^{n}\right)\right\| \\
& \lesssim\left\|m\left|M\left(B_{p}^{s, \alpha}\left(\mathbb{R}^{n}\right)\right)\left\|2^{-j\left(s-\frac{n}{p}\right)}\right\| \psi\left(2^{j \alpha} \cdot-l\right)\right| B_{p}^{s, \alpha}\left(\mathbb{R}^{n}\right)\right\| \\
& \lesssim\left\|m\left|M\left(B_{p}^{s, \alpha}\left(\mathbb{R}^{n}\right)\right)\left\|2^{-j\left(s-\frac{n}{p}\right)}\right\| \psi\left(2^{-j \alpha} \cdot\right)\right| B_{p}^{s, \alpha}\left(\mathbb{R}^{n}\right)\right\| \\
& \lesssim\left\|m\left|M\left(B_{p}^{s, \alpha}\left(\mathbb{R}^{n}\right)\right)\|\| \psi\right| B_{p}^{s, \alpha}\left(\mathbb{R}^{n}\right)\right\| \\
& \lesssim\left\|m \mid M\left(B_{p}^{s, \alpha}\left(\mathbb{R}^{n}\right)\right)\right\|
\end{aligned}
$$

for all $l \in \mathbb{Z}^{n}, j \in \mathbb{N}_{0}$, and hence,

$$
\begin{aligned}
\left\|m \mid B_{p, \text { selfs }}^{s, \alpha}\left(\mathbb{R}^{n}\right)\right\|_{\psi} & =\sup _{j \in \mathbb{N}_{0}, l \in \mathbb{Z}^{n}}\left\|\psi(\cdot-l) m\left(2^{-j \alpha} \cdot\right) \mid B_{p}^{s, \alpha}\left(\mathbb{R}^{n}\right)\right\| \\
& \lesssim\left\|m \mid M\left(B_{p}^{s, \alpha}\left(\mathbb{R}^{n}\right)\right)\right\| .
\end{aligned}
$$

We shall prove now the first inclusion in (i). Let $m \in B_{p, \text { selfs }}^{\sigma, \alpha}\left(\mathbb{R}^{n}\right)$ with $\sigma>s$. Let $f \in B_{p}^{s, \alpha}\left(\mathbb{R}^{n}\right)$ and let

$$
f=\sum_{j=0}^{\infty} \sum_{l \in \mathbb{Z}^{n}} \lambda_{j l} a_{j l}^{\alpha} \quad \text { with } \quad\left\|f\left|B_{p}^{s, \alpha}\left(\mathbb{R}^{n}\right)\|\sim\| \lambda\right| b_{p}\right\|
$$

be an optimal smooth atomic decomposition, where $a_{j l}^{\alpha}$ are anisotropic $(s, p)_{K^{-}}$ atoms with $K \geq \sigma+\alpha_{n}$. Then

$$
m f=\sum_{j=0}^{\infty} \sum_{l \in \mathbb{Z}^{n}} \lambda_{j l}\left(m a_{j l}^{\alpha}\right)
$$

and we wish to prove that, up to normalizing constants, $m a_{j l}^{\alpha}$ are anisotropic $(s, p)^{\sigma}$-atoms. The support condition is obvious:

$$
\operatorname{supp} m a_{j l}^{\alpha} \subset \operatorname{supp} a_{j l}^{\alpha} \subset d Q_{j l}^{\alpha}, \quad j \in \mathbb{N}_{0}, l \in \mathbb{Z}^{n} .
$$

If $l=0$ then we put $a_{j}^{\alpha}=a_{j 0}^{\alpha}$. Note that

$$
\operatorname{supp} a_{j}^{\alpha}\left(2^{-j \alpha} \cdot\right) \subset\left\{y:\left|y_{i}\right| \leq d / 2\right\}
$$

and we can assume that

$$
\psi(y)>0 \quad \text { if } \quad y \in\left\{x:\left|x_{i}\right| \leq d\right\} .
$$


Using Lemma 2.9, we have, for any $g \in B_{p}^{\sigma, \alpha}\left(\mathbb{R}^{n}\right)$,

$$
\begin{aligned}
\left\|a_{j}^{\alpha}\left(2^{-j \alpha} \cdot\right) \psi^{-1} g \mid B_{p}^{\sigma, \alpha}\left(\mathbb{R}^{n}\right)\right\| & \lesssim\left\|a_{j}^{\alpha}\left(2^{-j \alpha} \cdot\right) \psi^{-1}\left|C^{K, \alpha}\left(\mathbb{R}^{n}\right)\|\| g\right| B_{p}^{\sigma, \alpha}\left(\mathbb{R}^{n}\right)\right\| \\
& \lesssim 2^{-j\left(s-\frac{n}{p}\right)}\left\|g \mid B_{p}^{\sigma, \alpha}\left(\mathbb{R}^{n}\right)\right\|
\end{aligned}
$$

and hence

$$
\left\|a_{j}^{\alpha}\left(2^{-j \alpha} \cdot\right) \psi^{-1} \mid M\left(B_{p}^{\sigma, \alpha}\left(\mathbb{R}^{n}\right)\right)\right\| \lesssim 2^{-j\left(s-\frac{n}{p}\right)}, \quad j \in \mathbb{N}_{0} .
$$

By (52) and the homogeneity property we then get for $j \in \mathbb{N}_{0}$

$$
\begin{aligned}
\| m a_{j}^{\alpha} \mid & B_{p}^{\sigma, \alpha}\left(\mathbb{R}^{n}\right)\left\|\sim 2^{j\left(\sigma-\frac{n}{p}\right)}\right\| m\left(2^{-j \alpha} \cdot\right) a_{j}^{\alpha}\left(2^{-j \alpha} \cdot\right) \mid B_{p}^{\sigma, \alpha}\left(\mathbb{R}^{n}\right) \| \\
& \lesssim 2^{j\left(\sigma-\frac{n}{p}\right)}\left\|a_{j}^{\alpha}\left(2^{-j \alpha} \cdot\right) \psi^{-1}\left|M\left(B_{p}^{\sigma, \alpha}\left(\mathbb{R}^{n}\right)\right)\|\| m\left(2^{-j \alpha} \cdot\right) \psi\right| B_{p}^{\sigma, \alpha}\left(\mathbb{R}^{n}\right)\right\| \\
& \lesssim 2^{j(\sigma-s)}\left\|m\left(2^{-j \alpha} \cdot\right) \psi \mid B_{p}^{\sigma, \alpha}\left(\mathbb{R}^{n}\right)\right\| .
\end{aligned}
$$

In case of $a_{j l}^{\alpha}$ with $l \in \mathbb{Z}^{n}$ one would arrive at (53) with $a_{j l}^{\alpha}$ and $\psi(\cdot-l)$ instead of $a_{j}^{\alpha}$ and $\psi$, respectively. Hence

$$
\begin{aligned}
\left\|m a_{j l}^{\alpha} \mid B_{p}^{\sigma, \alpha}\left(\mathbb{R}^{n}\right)\right\| & \lesssim 2^{j(\sigma-s)} \sup _{j, l}\left\|m\left(2^{-j \alpha} \cdot\right) \psi(\cdot-l) \mid B_{p}^{\sigma, \alpha}\left(\mathbb{R}^{n}\right)\right\| \\
& =2^{j(\sigma-s)}\left\|m \mid B_{p, \text { selfs }}^{\sigma, \alpha}\left(\mathbb{R}^{n}\right)\right\|, \quad j \in \mathbb{N}_{0}, l \in \mathbb{Z}^{n},
\end{aligned}
$$

and therefore, $m a_{j l}^{\alpha}$ are anisotropic $(s, p)^{\sigma}$-atoms. By Theorem 4.3, in view of (49), $m f \in B_{p}^{\sigma, \alpha}\left(\mathbb{R}^{n}\right)$ and

$$
\begin{aligned}
\left\|m f \mid B_{p}^{\sigma, \alpha}\left(\mathbb{R}^{n}\right)\right\| & \lesssim\left\|\lambda\left|b_{p}\|\| m\right| B_{p, \text { selfs }}^{\sigma, \alpha}\left(\mathbb{R}^{n}\right)\right\| \\
& \sim\left\|f\left|B_{p}^{s, \alpha}\left(\mathbb{R}^{n}\right)\|\| m\right| B_{p, \text { selfs }}^{\sigma, \alpha}\left(\mathbb{R}^{n}\right)\right\|,
\end{aligned}
$$

which completes the proof of (i).

We prove (ii). Let $m \in B_{p, \text { selfs }}^{s, \alpha}\left(\mathbb{R}^{n}\right)$ and $p \leq 1$. It follows from (54) with $\sigma=s$ that

$$
\left\|m a_{j l}^{\alpha}\left|B_{p}^{s, \alpha}\left(\mathbb{R}^{n}\right)\|\lesssim\| m\right| B_{p, \text { selfs }}^{s, \alpha}\left(\mathbb{R}^{n}\right)\right\|, \quad j \in \mathbb{N}_{0}, l \in \mathbb{Z}^{n} .
$$

Since $B_{p}^{s, \alpha}\left(\mathbb{R}^{n}\right)$ is a $p$-Banach space, from (48) and using (49) and (55), we obtain

$$
\begin{aligned}
\left\|m f \mid B_{p}^{s, \alpha}\left(\mathbb{R}^{n}\right)\right\|^{p} & \leq \sum_{j=0}^{\infty} \sum_{l \in \mathbb{Z}^{n}}\left|\lambda_{j l}\right|^{p}\left\|m a_{j l}^{\alpha} \mid B_{p}^{s, \alpha}\left(\mathbb{R}^{n}\right)\right\|^{p} \\
& \lesssim\left\|\lambda\left|b_{p}\left\|^{p}\right\| m\right| B_{p, \text { selfs }}^{s, \alpha}\left(\mathbb{R}^{n}\right)\right\|^{p} \\
& \lesssim\left\|f\left|B_{p}^{s, \alpha}\left(\mathbb{R}^{n}\right)\left\|^{p}\right\| m\right| B_{p, \text { selfs }}^{s, \alpha}\left(\mathbb{R}^{n}\right)\right\|^{p}
\end{aligned}
$$


Hence $m \in M\left(B_{p}^{s, \alpha}\left(\mathbb{R}^{n}\right)\right)$ and, moreover, $B_{p, \text { selfs }}^{s, \alpha}\left(\mathbb{R}^{n}\right) \hookrightarrow M\left(B_{p}^{s, \alpha}\left(\mathbb{R}^{n}\right)\right)$. The other embedding follows from part (i).

The final part of this work is devoted to the question in which anisotropic function spaces the characteristic function $\chi_{\Omega}$ of the domain $\Omega$ in $\mathbb{R}^{n}$ is a pointwise multiplier.

Definition 5.5. Let $\alpha$ be an anisotropy according to (2) and let $\Gamma$ be a nonempty compact set in $\mathbb{R}^{n}$. Let $h: t \longmapsto h(t)$ be a positive monotonically non-decreasing function on the interval $(0,1]$. Then $\Gamma$ is called an anisotropic $h$-set if there is a finite Radon measure $\mu$ in $\mathbb{R}^{n}$ with

$$
\operatorname{supp} \mu=\Gamma \quad \text { and } \quad \mu\left(B^{\alpha}(\gamma, r)\right) \sim h(r), \quad \gamma \in \Gamma, 0<r \leq 1,
$$

where

$$
B^{\alpha}(\gamma, r)=\left\{x \in \mathbb{R}^{n}: \quad|x-\gamma|_{\alpha}<r\right\} .
$$

We say that the measure $\mu$ satisfies the anisotropic doubling condition if there is a constant $c>0$ such that

$$
\mu\left(B^{\alpha}(\gamma, 2 r)\right) \leq c \mu\left(B^{\alpha}(\gamma, r)\right), \quad \gamma \in \Gamma, \quad 0<r<1 .
$$

Let

$$
D_{\alpha}(x)=\operatorname{dist}_{\alpha}(x, \Gamma)=\inf _{y \in \Gamma}|x-y|_{\alpha}
$$

be the anisotropic distance of $x \in \mathbb{R}^{n}$ to $\Gamma$.

Theorem 5.6. Let $\Omega$ be a bounded domain in $\mathbb{R}^{n}$ and let $\alpha$ be an anisotropy according to (2). Moreover, let $0<p<\infty, \sigma\rangle \sigma_{p}$, and let $\Gamma=\partial \Omega$ be an anisotropic $h$-set according to Definition 5.5 with

$$
\sup _{j \in \mathbb{N}_{0}} \sum_{k=0}^{\infty} 2^{k \sigma p}\left(\frac{h\left(2^{-j}\right)}{h\left(2^{-j-k}\right)} 2^{-k n}\right)<\infty .
$$

Let $B_{p, \text { selfs }}^{\sigma, \alpha}\left(\mathbb{R}^{n}\right)$ be the space introduced in Definition 5.1. Then

$$
\chi_{\Omega} \in B_{p, \text { selfs }}^{\sigma, \alpha}\left(\mathbb{R}^{n}\right) .
$$

Proof: The proof is based upon ideas found in [12, Theorem 3]. It simplifies the argument, and causes no loss of generality, to assume diam $\Omega<1$. We define

$$
\Omega^{k}=\left\{x \in \Omega: 2^{-k-2} \leq \operatorname{dist}_{\alpha}(x, \Gamma) \leq 2^{-k}\right\}, \quad k \in \mathbb{N}_{0} .
$$


Moreover, let

$$
\left\{\varphi_{l}^{k, \alpha}: k \in \mathbb{N}_{0} ; l=1, \ldots, M_{k}\right\} \subset C_{0}^{\infty}(\Omega)
$$

be an anisotropic resolution of unity,

$$
\sum_{k \in \mathbb{N}_{0}} \sum_{l=1}^{M_{k}} \varphi_{l}^{k, \alpha}(x)=1, \quad \text { if } \quad x \in \Omega
$$

with

$$
\operatorname{supp} \varphi_{l}^{k, \alpha} \subset\left\{x:\left|x-x_{l}^{k}\right|_{\alpha} \leq 2^{-k}\right\} \subset \Omega^{k}
$$

and

$$
\left|\mathrm{D}^{\gamma} \varphi_{l}^{k, \alpha}(x)\right| \lesssim 2^{\gamma \alpha k} \quad \text { for } \quad \gamma \alpha \leq K, \quad x \in \mathbb{R}^{n}, K \in \mathbb{N} \text { with } K \geq \sigma+\alpha_{n} .
$$

It turns out that such an anisotropic resolution of unity exists. See [11, Section 7.5] for discussion of this technical point in the isotropic case. We now estimate the minimal number $M_{k}$ in (60). Combining the fact that the measure $\mu$ satisfies the doubling condition (57) together with (56) we arrive at

$$
M_{k} h\left(2^{-k}\right) \lesssim 1, \quad k \in \mathbb{N}_{0}
$$

Clearly, (60) can be rewritten in the form

$$
\chi_{\Omega}(x)=\sum_{k=0}^{\infty} 2^{k\left(\sigma-\frac{n}{p}\right)} \sum_{l=0}^{M_{k}} 2^{-k\left(\sigma-\frac{n}{p}\right)} \varphi_{l}^{k, \alpha}(x), \quad x \in \mathbb{R}^{n},
$$

where $2^{-k\left(\sigma-\frac{n}{p}\right)} \varphi_{l}^{k, \alpha}$ are anisotropic $(\sigma, p)_{K^{-a t o m s}}$ according to Definition 2.5. Furthermore, we obtain

$$
\left\|\chi_{\Omega} \mid B_{p}^{\sigma, \alpha}\left(\mathbb{R}^{n}\right)\right\|^{p} \leq \sum_{k=0}^{\infty} 2^{k\left(\sigma-\frac{n}{p}\right) p} M_{k} \lesssim \sum_{k=0}^{\infty} 2^{k \sigma p}\left(\frac{2^{-k n}}{h\left(2^{-k}\right)}\right)<\infty
$$

This shows that $\chi_{\Omega} \in B_{p}^{\sigma, \alpha}\left(\mathbb{R}^{n}\right)$. We now prove that $\chi_{\Omega} \in B_{p \text {,selfs }}^{\sigma, \alpha}\left(\mathbb{R}^{n}\right)$. We consider the non-negative function $\psi \in C^{\infty}\left(\mathbb{R}^{n}\right)$ with (43) and (44). By the definition of anisotropic selfsimilar spaces, it suffices to consider

$$
\chi_{\Omega}\left(2^{-j \alpha} \cdot\right) \psi
$$

assuming in addition that $0 \in 2^{j \alpha} \Gamma=\left\{2^{j \alpha} \gamma=\left(2^{j \alpha_{1}} \gamma_{1}, \ldots, 2^{j \alpha_{n}} \gamma_{n}\right): \quad \gamma \in \Gamma\right\}$, $j \in \mathbb{N}_{0}$. Let $\mu^{j}$ be the image measure of $\mu$ with respect to the dilations 
$y \longmapsto 2^{j \alpha} y$. Then we obtain

$$
\mu^{j}\left(B^{\alpha}(0, \sqrt{n}) \cap 2^{j \alpha} \Gamma\right) \sim h\left(2^{-j}\right), \quad j \in \mathbb{N}_{0} .
$$

We use the same argument as above to $B^{\alpha}(0, \sqrt{n}) \cap 2^{j \alpha} \Omega$ and $B^{\alpha}(0, \sqrt{n}) \cap 2^{j \alpha} \Gamma$. Hence, we again have

$$
M_{k}^{j} h\left(2^{-j-k}\right) \lesssim h\left(2^{-j}\right), \quad j \in \mathbb{N}_{0}, \quad k \in \mathbb{N}_{0},
$$

which completes the proof.

\title{
References
}

[1] A. Caetano, S. Lopes, H. Triebel, A homogeneity property for Besov spaces, J. Funct. Spaces Appl. (to appear).

[2] S. Dachkovski, Anisotropic function spaces and related semi-linear hypoelliptic equations, Math. Nachr. 248/249 (2003), 40-61.

[3] W. Farkas, Atomic and subatomic decompositions in anisotropic function spaces, Math. Nachr. 209 (2000), 83-113.

[4] S. D. Moura, J. S. Neves, M. Piotrowski, Growth envelopes of anisotropic function spaces, $Z$. Anal. Anwendungen (to appear).

[5] T. Runst, W. Sickel, Sobolev spaces of fractional order, Nemytskii operators, and nonlinear partial differential operators, DeGruyter Verlag, Berlin, 1996.

[6] E. M. Stein, Harmonic analysis: real-variable methods, orthogonality, and oscillatory integrals, Princeton University Press, Princeton, NJ, 1993.

[7] H.-J. Schmeisser, H. Triebel, Topics in Fourier Analysis and Function Spaces, John Wiley \& Sons, Chichester, 1987.

[8] E. Tamási, Anisotropic Besov spaces and approximation numbers of traces on related fractal sets, Rev. Mat. Complutense 19 (2006), 297-321.

[9] H. Triebel, Fourier Analysis and Function Spaces, Teubner, Leipzig, 1977.

[10] H. Triebel, Theory of Function Spaces II, Birkhäuser, Basel, 1992.

[11] H. Triebel, The Structure of Functions, Birkhäuser, Basel, 2001.

[12] H. Triebel, Non-smooth atoms and pointwise multipliers in function spaces, Ann. Mat. Pura Appl. 182 (2003), 457-486 .

[13] H. Triebel, Theory of Function Spaces III, Birkhäuser, Basel, 2006.

\author{
Susana D. MOURA \\ Department of Mathematics, University of Coimbra, Apartado 3008, 3001-454 Coimbra, \\ PORTUGAL \\ E-mail address: smpsd@mat.uc.pt \\ IWONA PIOTROWSKA \\ Mathematical Institute, Friedrich-Schiller-University JenA, D-07737 Jena, Germany \\ E-mail address: iwona@minet.uni-jena.de \\ MARiUSZ PiOTROWSKI \\ Department of Mathematics, University of Coimbra, Apartado 3008, 3001-454 Coimbra, \\ PORTUGAL \\ E-mail address: marian@mat.uc.pt
}

